\title{
BILINEAR LITTLEWOOD-PALEY FOR CIRCLE AND TRANSFERENCE
}

\author{
Parasar Mohanty and Saurabh Shrivastava
}

Abstract

In this paper we have obtained the boundedness of bilinear Littlewood-Paley operators on the circle group $\mathbb{T}$ by using appropriate transference techniques. In particular, bilinear analogue of Carleson's Littlewood-Paley result for all possible indices has been obtained. Also, we prove some bilinear analogues of de Leeuw's results concerning multipliers of $\mathbb{R}^{n}$.

\section{Introduction}

Let $m$ be a bounded measurable function defined on $\mathbb{R}^{2 n}$. Consider the bilinear operator $T$ associated with the symbol $m$

$$
T(f, g)(x)=\int_{\mathbb{R}^{n}} \int_{\mathbb{R}^{n}} \hat{f}(\xi) \hat{g}(\eta) m(\xi, \eta) e^{2 \pi i x \cdot(\xi+\eta)} d \xi d \eta,
$$

defined for functions $f$ and $g$ belonging to the Schwartz space $\mathcal{S}\left(\mathbb{R}^{n}\right)$. We say that $m$ is a bilinear multiplier on $\mathbb{R}^{n}$ for the triplet $\left(p_{1}, p_{2}, p_{3}\right)$, where $1 \leq p_{1}, p_{2} \leq \infty$ and $\frac{1}{p_{1}}+\frac{1}{p_{2}}=\frac{1}{p_{3}}$, if the operator $T$ extends to a bounded operator from $L^{p_{1}}\left(\mathbb{R}^{n}\right) \times L^{p_{2}}\left(\mathbb{R}^{n}\right)$ into $L^{p_{3}}\left(\mathbb{R}^{n}\right)$, i.e.

$$
\|T(f, g)\|_{p_{3}} \leq c\|f\|_{p_{1}}\|g\|_{p_{2}},
$$

where $c$ is a constant independent of functions $f$ and $g$. In a similar fashion we can define bilinear multipliers on the $n$-dimensional torus $\mathbb{T}^{n}$.

After Lacey and Thiele's work [14], [15] on bilinear Hilbert transform, where $m(\xi, \eta)=-i \operatorname{sgn}(\xi-\eta)$ for $\xi, \eta \in \mathbb{R}$, the study of bilinear multipliers got lot of attention. The set of all bilinear multipliers on $\mathbb{R}^{n}$ for the triplet $\left(p_{1}, p_{2}, p_{3}\right)$ will be denoted by $M_{p_{1}, p_{2}}^{p_{3}}\left(\mathbb{R}^{n}\right)$. For $p_{3} \geq 1$, $M_{p_{1}, p_{2}}^{p_{3}}\left(\mathbb{R}^{n}\right)$ becomes a Banach space under the operator norm, whereas

2010 Mathematics Subject Classification. 42A45, 42B15, 42B25, 42A75.

Key words. Bilinear multipliers, Littlewood-Paley square functions, transference methods. 
for $0<p_{3}<1$ it forms a quasi Banach space. For details we would like to refer the interested reader to the article of Grafakos and Torres [12].

In the theory of linear multipliers, the smooth and non-smooth Littewood-Paley square functions play important roles. Let us first recall some classical results concerning square functions from linear multiplier theory.

Let $\left\{I_{n}\right\}_{n \in \mathbb{Z}}$ be a sequence of disjoint intervals in $\mathbb{R}$. Then the associated non-smooth Littlewood-Paley square function is defined as

$$
\mathcal{T} f(x):=\left(\sum_{n \in \mathbb{Z}}\left|\mathcal{T}_{I_{n}}(f)(x)\right|^{2}\right)^{\frac{1}{2}}, \quad f \in \mathcal{S}(\mathbb{R}),
$$

where $\mathcal{T}_{I_{n}}$ is the linear multiplier operator given by $\widehat{\mathcal{T}_{n} f}(\xi)=\chi_{I_{n}}(\xi) \hat{f}(\xi)$. The smooth analogue of the operator $\mathcal{T}$ is defined by considering compactly supported smooth functions $\phi_{n}$ instead of $\chi_{I_{n}}$. It is well-known that the $L^{p}$ boundedness properties of the operator $\mathcal{T}$ can be derived from boundedness of a suitable smooth square function with some vector valued arguments. The celebrated result of Littlewood and Paley [10] states that if $I_{n}=\left(-2^{n+1},-2^{n}\right] \cup\left[2^{n}, 2^{n+1}\right), n \in \mathbb{Z}$, then for $1<p<\infty$ and $f \in \mathcal{S}(\mathbb{R})$, we have

$$
\left\|\left(\sum_{n \in \mathbb{Z}}\left|\mathcal{T}_{I_{n}}(f)\right|^{2}\right)^{\frac{1}{2}}\right\|_{L^{p}(\mathbb{R})} \cong\|f\|_{L^{p}(\mathbb{R})} .
$$

Here the notation ' $A \cong B$ ' means that there are two constants $c_{1}$, $c_{2}$ such that $c_{1} A \leq B \leq c_{2} A$. Later, in the year 1967, Carleson [5] considered the Littlewood-Paley square function associated with the sequence $I_{n}=[n, n+1), n \in \mathbb{Z}$, and proved that for $2 \leq p<\infty$, there exists a constant $C_{p}$ such that for all $f \in \mathcal{S}(\mathbb{R})$, we have

$$
\left\|\left(\sum_{n \in \mathbb{Z}}\left|\mathcal{T}_{I_{n}}(f)\right|^{2}\right)^{\frac{1}{2}}\right\|_{L^{p}(\mathbb{R})} \leq C_{p}\|f\|_{L^{p}(\mathbb{R})} .
$$

In the year of 1985, Rubio de Francia [18] proved above inequality (4) for the case of arbitrary sequence of disjoint intervals in $\mathbb{R}$. In both the previous results $p \geq 2$ is an optimal condition.

Our interest here is to study some bilinear analogues of these questions on the circle group $\mathbb{T}$. Lacey [13] introduced the bilinear LittlewoodPaley square function. Let $\left\{I_{l}\right\}_{l \in \mathbb{Z}}$ be a sequence of disjoint intervals 
in $\mathbb{R}$. Then the associated non-smooth bilinear Littlewood-Paley operator is defined as

$$
S(f, g)(x):=\left(\sum_{l \in \mathbb{Z}}\left|S_{I_{l}}(f, g)(x)\right|^{2}\right)^{\frac{1}{2}}, \quad f, g \in \mathcal{S}(\mathbb{R}),
$$

where $S_{I_{l}}$ is the bilinear multiplier operator associated with the symbol $\chi_{I_{l}}(\xi-\eta)$. The smooth version is defined naturally replacing $\chi_{I_{l}}(\xi-$ $\eta$ ) by smooth function $\phi_{l}(\xi-\eta)$. We are interested in $L^{p}$ boundedness properties of these bilinear square functions. We say that the operator $S$ is bounded for triplet $\left(p_{1}, p_{2}, p_{3}\right)$ satisfying Hölder condition $\frac{1}{p_{1}}+\frac{1}{p_{2}}=\frac{1}{p_{3}}$, if

$$
\|S(f, g)\|_{p_{3}} \leq C\|f\|_{p_{1}}\|g\|_{p_{2}} .
$$

Lacey [13] proved the boundedness of a bilinear analogue of smooth Carleson's Littlewood-Paley operator on $\mathbb{R}$ for $p_{3}=2$. Later, Mohanty and Shrivastava [17] extended Lacey's results for $p_{3}>\frac{4}{3}$ and $p_{1}, p_{2}>2$. The problem for non-smooth version was addressed by Bernicot [1]. He proved the boundedness of a version of non-smooth bilinear LittlewoodPaley operator. In particular, he obtained the bilinear analogue of Carleson's Littlewood-Paley inequality (4) on $\mathbb{R}$ in the local $L^{2}$ case, i.e. $2<p_{1}, p_{2}, p_{3}^{\prime}<\infty$.

In $\S 2$ we have considered bilinear Littlewood-Paley square functions on circle group $\mathbb{T}$. Let $\left\{\tilde{I}_{l}\right\}_{l \in \mathbb{Z}}$ be a sequence of disjoint intervals in $\mathbb{Z}$ and $\tilde{S}_{l}(f, g)$ be the bilinear multiplier operator associated with the symbol $\chi_{\tilde{I}_{l}}(n-m)$, i.e., for $f, g \in \mathcal{P}(\mathbb{T})$, trignometric polynomials on $\mathbb{T}$,

$$
\tilde{S}_{l}(f, g)(x)=\sum_{n, m} \hat{f}(n) \hat{g}(m) \chi_{\tilde{I}_{l}}(n-m) e^{2 \pi i x(n+m)} .
$$

The bilinear Littlewood-Paley square function associated with this sequence is given by

$$
\tilde{S}(f, g)(x)=\left(\sum_{l \in \mathbb{Z}}\left|\tilde{S}_{l}(f, g)(x)\right|^{2}\right)^{\frac{1}{2}}, \quad x \in \mathbb{T} .
$$

Observe that in the linear case Carleson's theorem on $\mathbb{T}$ is trivial as the interval $[n, n+1)$ is just singleton $\{n\}$. In this case the linear operators $\tilde{T}_{n}$ satisfy, $\tilde{T}_{n}(f)(x)=\left(\chi_{\tilde{I}_{n}} \hat{f}\right)(x)=\hat{f}(n) e^{2 \pi i n x}$, for $x \in \mathbb{T}$. Then the associated Littlewood-Paley square function $\tilde{T}$ becomes

$$
\left(\sum_{n \in \mathbb{Z}}\left|\tilde{T}_{n}(f)(x)\right|^{2}\right)^{\frac{1}{2}}=\left(\sum_{n \in \mathbb{Z}}|\hat{f}(n)|^{2}\right)^{\frac{1}{2}}=\|f\|_{2} .
$$


Hence, for $2 \leq p$

$$
\left\|\left(\sum_{n \in \mathbb{Z}}\left|\tilde{T}_{n}(f)\right|^{2}\right)^{\frac{1}{2}}\right\|_{p}=\|f\|_{2} \leq\|f\|_{p} .
$$

But this is no longer true in the bilinear setting. As in the bilinear case one can easily see that for $\tilde{I}_{l}=\{l\}$, we have following description of the associated bilinear operator

$$
\tilde{S}_{l}(f, g)(x)=e^{2 \pi i l x}\left(e^{-2 \pi i l .} f * g\right)(2 x) .
$$

Like the linear case by estimating the $L^{2}(\mathbb{T})$ norm of bilinear Carleson's Littlewood-Paley square function directly and using the nested property of $L^{p}(\mathbb{T})$ spaces (see $\S 2$ for precise arguments), we can prove boundedness of this square function only for exponents satisfying $2 \leq p_{1}, p_{2}, p_{3}^{\prime} \leq$ $\infty$. So, for other exponents bilinear Carleson's Littlewood-Paley square function becomes an interesting object. In $\S 2$ we prove the boundedness of this operator for all possible exponents. Also, we prove a bilinear analogue of Bernicot's result $[\mathbf{1}]$ on $\mathbb{T}$. We have used suitable vector valued transference techniques to obtain these results.

In $\S 3$ and $\S 4$ we continue with the transference techniques and prove some bilinear analogues of de Leeuw's results concerning transferring linear multipliers among Euclidean groups. In order to state one of de Leeuw's result we first provide the definition for Besicovitch spaces of almost periodic functions. For $0<p<\infty$, Besicovitch space is denoted by $B^{p}(\mathbb{R})$ and is defined as the closure of trigonometric polynomials with respect to the semi-norm

$$
\|f\|_{B^{p}(\mathbb{R})}=\lim _{T \rightarrow \infty}\left(\frac{1}{2 T} \int_{-T}^{T}|f(x)|^{p} d x\right)^{\frac{1}{p}} .
$$

Note that there are non-zero functions $f$ with $\|f\|_{B^{p}(\mathbb{R})}=0$. So one has to quotient out these functions to get a norm for $1 \leq p<\infty$ and a quasi norm for $0<p<1$. We shall use the same notation $\|\cdot\|_{B^{p}(\mathbb{R})}$ for this norm and quasi norm as well. When $p=\infty$, we use $B^{\infty}(\mathbb{R})$ to denote the corresponding space and it is defined as the closure of trigonometric polynomials with respect to the $L^{\infty}(\mathbb{R})$ norm. For an almost periodic function $f$, its Bohr transform at the point $\lambda \in \mathbb{R}$ is defined as

$$
\hat{f}(\lambda)=\lim _{T \rightarrow \infty} \frac{1}{2 T} \int_{-T}^{T} f(x) e^{-2 \pi i \lambda x} d x .
$$


Note that here we are using the same notation for Bohr transform and Fourier transform.

We say that a bounded function $m(t, s)$ is an almost periodic bilinear multiplier for the triplet $\left(p_{1}, p_{2}, p_{3}\right)$ if the operator $B$ initially defined for the trigonometric polynomials $f$ and $g$ by

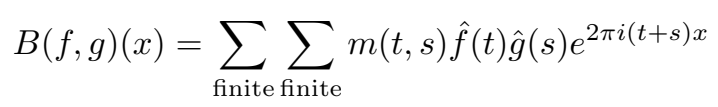

extends to a bounded operator form $B^{p_{1}}(\mathbb{R}) \times B^{p_{2}}(\mathbb{R})$ into $B^{p_{3}}(\mathbb{R})$, i.e.

$$
\|B(f, g)\|_{B^{p_{3}(\mathbb{R})}} \leq C\|f\|_{B^{p_{1}(\mathbb{R})}}\|g\|_{B^{p_{2}(\mathbb{R})}} .
$$

Let $\mathcal{M}_{p_{1}, p_{2}}^{p_{3}}(\mathbb{R})$ denote the space of all almost periodic bilinear multipliers for the triplet $\left(p_{1}, p_{2}, p_{3}\right)$.

In [7] de Leeuw proved that multipliers on $L^{p}(\mathbb{R})$ are precisely the ones which are multipliers on $B^{p}(\mathbb{R})$ and vice versa. The bilinear analogue of this has been addressed by Blasco [2], but with the condition $p_{3} \geq 1$. However the methods employed by Blasco do not extend to the case $p_{3}<$ 1. We extend this result to the full possible range of exponents and our method is significantly different from $[\mathbf{2}]$.

In the same paper de Leeuw proved that if a continuous function $m$ is an $L^{p}\left(\mathbb{R}^{n}\right)$ multiplier, then $\left.m\right|_{\mathbb{R}^{k}}$ is an $L^{p}\left(\mathbb{R}^{k}\right)$ multiplier for $k<n$. A bilinear analogue of this result is proved by Diestel and Grafakos [8] for $p_{3} \geq 1$. Again, in $\S 4$, we have extended this result to the entire range of the exponents.

\section{Bilinear Littlewood-Paley square functions on $\mathbb{T}$}

In this section we address bilinear Littlewood-Paley square functions on $\mathbb{T}$. Let $\left\{\tilde{I}_{l}\right\}_{l \in \mathbb{Z}}$ be a sequence of disjoint intervals of equal lengths in $\mathbb{Z}$. Suppose $\tilde{I}_{l}=\left[a_{l}, b_{l}\right)$ and $b_{l}-a_{l}=L$ for all $l \in \mathbb{Z}$. For $f, g \in \mathcal{P}(\mathbb{T})$, consider

$$
\begin{aligned}
\tilde{S}_{l}(f, g)(x) & =\sum_{n, m} \hat{f}(n) \hat{g}(m) \chi_{\tilde{I}_{l}}(n-m) e^{2 \pi i x(n+m)} \\
& =\sum_{j=0}^{L-1} \sum_{m} \hat{f}\left(m+a_{l}+j\right) \hat{g}(m) e^{2 \pi i x\left(2 m+a_{l}+j\right)} \\
& =\sum_{j=0}^{L-1} e^{2 \pi i x\left(a_{l}+j\right)} F_{l, j}(x),
\end{aligned}
$$


where $F_{l, j}(x)=\left(f(.) e^{-2 \pi i\left(a_{l}+j\right)} * g\right)(2 x)$.

$$
\begin{aligned}
\|\tilde{S}(f, g)\|_{2}^{2} & =\int_{0}^{1} \sum_{l \in \mathbb{Z}}\left|\tilde{S}_{l}(f, g)(x)\right|^{2} d x \\
& =\int_{0}^{1} \sum_{l \in \mathbb{Z}}\left|\sum_{j=0}^{L-1} e^{2 \pi i x\left(a_{l}+j\right)} F_{l, j}(x)\right|^{2} d x \\
& \leq\left(\sum_{j=0}^{L-1}\left(\int_{0}^{1} \sum_{l \in \mathbb{Z}}\left|F_{l, j}(x)\right|^{2} d x\right)^{\frac{1}{2}}\right)^{2} \\
& =\left(\sum_{0}^{L-1}\left(\sum_{l \in \mathbb{Z}} \sum_{n \in \mathbb{Z}}\left|\hat{f}\left(n+a_{l}+j\right) \hat{g}(n)\right|^{2}\right)^{\frac{1}{2}}\right)^{2} \\
& \leq\left(L\|f\|_{2}\|g\|_{2}\right)^{2} .
\end{aligned}
$$

Here we have used Minkowski's inequality and Plancherel theorem. Now using the fact that $\|\cdot\|_{L^{s}(\mathbb{T})} \leq\|\cdot\|_{L^{t}(\mathbb{T})}$ for $1 \leq s \leq t \leq \infty$, we get

$$
\|\tilde{S}(f, g)\|_{p_{3}} \leq L\|f\|_{p_{1}}\|g\|_{p_{2}},
$$

where $2 \leq p_{1}, p_{2} \leq \infty$ and $1 \leq p_{3} \leq 2$. In particular, bilinear analogue of Carleson's Littlewood-Paley result is obtained for $2 \leq p_{1}, p_{2} \leq \infty$ and $1 \leq p_{3} \leq 2$, as mentioned in the Introduction.

At this point there are two issues. The first one is to get boundedness of bilinear Carleson's Littlewood-Paley square function for remaining exponents, i.e. for $p_{3}>2$ and the second is to get rid of the dependency on the length $L$ in inequality (10). The first issue is resolved completely by transferring a result of Mohanty and Shrivastava [17] concerning smooth bilinear square function on $\mathbb{R}$ to $\mathbb{T}$. The second issue is dealt with for the sequences of intervals which are of equal lengths and equi-distant, by transferring Bernicot's [1] non-smooth bilinear square functions on $\mathbb{R}$ to $\mathbb{T}$. For both these purposes we use the suitable transference techniques adapted to vector valued bilinear settings.

2.1. Transference. Using Khintchine's inequality, observe that an equivalent formulation for the boundedness of the square functions can 
be given with the following expression

$$
\left\|\sum_{k} r_{k} \tilde{S}_{k}(f, g)\right\|_{L_{L^{p_{3}[0,1]}}^{p_{3}(\mathbb{T})}} \leq C\|f\|_{L^{p_{1}(\mathbb{T})}}\|g\|_{L^{p_{2}(\mathbb{T})}},
$$

where $r_{k}$ 's are Radamacher functions and $C$ is a constant independent of $f$ and $g$. This formulation of the problem motivates us to look for the following vector valued transference result. The linear version of this result can be found in $[\mathbf{9}]$. Since the proof of this theorem is very similar to the original proof of general transference theorem of Coifman and Weiss [6], we skip the proof here.

Theorem 2.1. Let $G$ be a locally compact amenable group. Let $X_{1}$, $X_{2}, X_{3}$ and $\tilde{X}_{3}$ be Banach spaces such that $X_{1} X_{2} \subseteq X_{3}$. Let $k$ be a compactly supported function in $L_{\mathcal{B}\left(X_{3}, \tilde{X}_{3}\right)}^{1}(G)$. Assume that there exist strongly continuous representations $R^{1}, R^{2}, R^{3}$ and $\tilde{R}^{3}$ of the group $G$ such that

(1) For every $u \in G$, we have $R_{u}^{i} \in \mathcal{B}\left(X_{i}\right)$ for $i=1,2,3$, and $\tilde{R}_{u}^{3} \in$ $\mathcal{B}\left(\tilde{X}_{3}\right)$.

(2) There exists $C>0$ such that $\left\|R_{u}^{i}\right\|_{\mathcal{B}\left(X_{i}\right)} \leq C, i=1,2,3$, and $\left\|\tilde{R}_{u}^{3}\right\|_{\mathcal{B}\left(\tilde{X}_{3}\right)} \leq C$ for all $u \in G$.

(3) $R^{3}$ and $\tilde{R}^{3}$ intertwine $k$ in the sense that

$$
\tilde{R}_{v}^{3} k(u)\left(x_{3}\right)=k(u) R_{v}^{3}\left(x_{3}\right), \quad u, v \in G, x_{3} \in X_{3} .
$$

(4) For $u, v, w \in G, x_{1} \in X_{1}$ and $x_{2} \in X_{2}$

$$
R_{w}^{3}\left(R_{u}^{1} x_{1} R_{v}^{2} x_{2}\right)=R_{w u}^{1} x_{1} R_{w v}^{2} x_{2} .
$$

Suppose that the bilinear operator

$$
T(f, g)(v)=\int_{G} k(u)\left(f\left(v u^{-1}\right) g(v u)\right) d u
$$

is bounded from $L_{X_{1}}^{p_{1}}(G) \times L_{X_{2}}^{p_{2}}(G)$ into $L_{\tilde{X}_{3}}^{p_{3}}(G)$ for Hölder related triplet $\left(p_{1}, p_{2}, p_{3}\right)$ with $p_{3} \geq 1$. Then the transferred operator

$$
T_{k}\left(x_{1}, x_{2}\right)=\int_{G} k(u)\left(R_{u^{-1}}^{1} x_{1} R_{u}^{2} x_{2}\right) d u
$$

is bounded from $X_{1} \times X_{2}$ into $\tilde{X}_{3}$. Moreover the operator norm of the transferred operator $T_{k}$ satisfies

$$
\left\|T_{k}\right\| \leq C N_{p_{1}, p_{2}, p_{3}}(k)
$$

where $N_{p_{1}, p_{2}, p_{3}}(k)$ is the operator norm of the original operator $T$. 
We will use the above transference theorem to address both the issues described earlier.

Take $G=\mathbb{R}, X_{i}=L^{p_{i}}(\mathbb{T})$ for $i=1,2,3$ and $\tilde{X}_{3}=L_{L^{p_{3}[0,1]}}^{p_{3}}(\mathbb{T})$. For $u \in \mathbb{R}$, define $R_{u}^{i}$ on $L^{p_{i}}(\mathbb{T})$ by $R_{u}^{i}\left(f_{i}\right)\left(e^{2 \pi i x}\right)=R_{u}\left(f_{i}\right)\left(e^{2 \pi i x}\right)=$ $f_{i}\left(e^{2 \pi i(u+x)}\right)$ for $f_{i} \in L^{p_{i}}(\mathbb{T}), i=1,2,3$ and $\tilde{R}_{u}^{3}$ on $L_{L^{p_{3}[0,1]}}^{p_{3}}(\mathbb{T})$ by $\tilde{R}_{u}^{3} g\left(e^{2 \pi i x}\right)=g\left(e^{2 \pi i(u+x)}\right)$ for $g \in L_{L^{p_{3}[0,1]}}^{p_{3}}(\mathbb{T})$. It can be easily verified that $R^{i}$ and $\tilde{R}^{3}$ are uniformly bounded representations of $\mathbb{R}$ on the respective spaces, as these are just the translation operators. Thus conditions (1), (2) and (4) of Theorem 2.1 are satisfied in this setting.

Let $k_{j} \in L^{1}(\mathbb{R})$ have compact support. For $u \in \mathbb{R}$ define $K(u) f=$ $\left(\sum_{j=1}^{N} r_{j}(.) k_{j}(u)\right) f$. With this definition of $K$ we observe that

$$
\tilde{R}_{v}^{3} K(u) f\left(e^{2 \pi i x}\right)=K(u) f\left(e^{2 \pi i(x+v)}\right)=K(u) R_{v}^{3} f\left(e^{2 \pi i x}\right), \quad u, v \in \mathbb{R} .
$$

Thus, condition (3) of Theorem 2.1 is also satisfied.

For $F, G \in \mathcal{S}(\mathbb{R})$ consider the operator

$$
\sum_{j=1}^{N} r_{j}(.) S_{\hat{k}_{j}}(F, G)(x)=\sum_{j=1}^{N} r_{j}(.) \int_{\mathbb{R}} \int_{\mathbb{R}} \hat{F}(\xi) \hat{G}(\eta) \hat{k}_{j}(\xi-\eta) e^{2 \pi i x(\xi+\eta)} d \xi d \eta \text {. }
$$

Then the transferred operator will look like

$$
\sum_{j=1}^{N} r_{j}(.) \tilde{S}_{\left.\hat{k}_{j}\right|_{\mathbb{Z}}}(f, g)(x)=\sum_{j=1}^{N} r_{j}(.) \sum_{n} \sum_{m} \hat{f}(n) \hat{g}(m) \hat{k}_{j}(n-m) e^{2 \pi i x(n+m)} \text {. }
$$

As an application of the Theorem 2.1 one can prove that if

$$
\left\|\sum_{j=1}^{N} r_{j} S_{\hat{k}_{j}}(F, G)\right\|_{L_{L^{p_{3}[0,1]}}^{p_{3}}(\mathbb{R})} \leq C\|F\|_{L^{p_{1}(\mathbb{R})}}\|G\|_{L^{p_{2}(\mathbb{R})},}
$$

then

$$
\left\|\sum_{j=1}^{N} r_{j} \tilde{S}_{\hat{k}_{j \mid \mathbb{Z}}}(f, g)\right\|_{L_{L^{p_{3}[0,1]}}^{p_{3}}(\mathbb{T})} \leq C\|f\|_{L^{p_{1}(\mathbb{T})}}\|g\|_{L^{p_{2}(\mathbb{T})}}
$$

Further, one can get inequality (14) from inequality (13) for normalized symbols $m_{j}$ in place of $\hat{k}_{j}$ by using standard approximation techniques (see [6]). A bounded measurable function $m$ is said to be normalized if

$$
m^{n}(x)=m * \frac{1}{2 n}\left(\chi_{[-n, n]} * \chi_{[-n, n]}\right)^{\wedge}(x) \rightarrow m(x) \quad \forall x \in \mathbb{R} \text { as } n \rightarrow \infty .
$$


For normalized symbols $m_{j}$, we define $k_{j}^{n}=h_{n} * \check{m}_{j}^{n}$, where $h_{n} \in$ $C_{c}^{\infty}(\mathbb{R})$ with $\hat{h_{n}}(x) \rightarrow 1$ as $n \rightarrow \infty$ and $\left\|h_{n}\right\|_{1}=1$. Observe that $k_{j}^{n} \in L^{1}(\mathbb{R})$ with compact support. Hence applying Theorem 2.1 we get that if

$$
\left\|\sum_{j=1}^{N} r_{j} S_{\hat{k}_{j}^{n}}(F, G)\right\|_{L_{L^{p_{3}[0,1]}}^{p_{3}}(\mathbb{R})} \leq C\|F\|_{L^{p_{1}(\mathbb{R})}}\|G\|_{L^{p_{2}(\mathbb{R})} .} .
$$

Then, we get the transferred inequality

$$
\left\|\sum_{j=1}^{N} r_{j} \tilde{S}_{\hat{k}_{j}^{n} \mid \mathbb{Z}}(f, g)\right\|_{L_{L^{p_{3}[0,1]}}^{p_{3}}(\mathbb{T})} \leq C\|f\|_{L^{p_{1}(\mathbb{T})}}\|g\|_{L^{p_{2}(\mathbb{T})}},
$$

where $C$ is the same constant as in inequality (15).

As $p_{3}>1$, by applying duality and dominated convergence theorem we can conclude that

$$
\left\|\sum_{j=1}^{N} r_{j} \tilde{S}_{m_{j} \mid \mathbb{Z}}(f, g)\right\|_{L_{L^{p_{3}[0,1]}}^{p_{3}}(\mathbb{T})} \leq C\|f\|_{L^{p_{1}(\mathbb{T})}}\|g\|_{L^{p_{2}(\mathbb{T})}} .
$$

Now we will prove bilinear Carleson's theorem on $\mathbb{T}$ for $p_{3}>2$ by transferring the bounds from the following known result.

Theorem $2.2([\mathbf{1 7}])$. Let $m \in \mathcal{S}(\mathbb{R})$. For $l \in \mathbb{Z}$, define $m_{l}(\xi)=m(\xi-l)$ and let $S_{m_{l}}$ be the bilinear multiplier operator associated with $m_{l}$. Then for $2<p_{1}, p_{2} \leq \infty$ and $4 / 3<p_{3} \leq \infty$ satisfying $\frac{1}{p_{1}}+\frac{1}{p_{2}}=\frac{1}{p_{3}}$, we have

$$
\left\|\left(\sum_{l \in \mathbb{Z}}\left|S_{m_{l}}(f, g)\right|^{2}\right)^{1 / 2}\right\|_{L^{p_{3}(\mathbb{R})}} \leq C\|f\|_{L^{p_{1}(\mathbb{R})}}\|g\|_{L^{p_{2}(\mathbb{R})}} .
$$

We now choose function $m$ suitably to get the desired conclusion. For this let $m$ be a $C_{c}^{\infty}(\mathbb{R})$ function such that $m=1$ on $\left[-\frac{1}{4}, \frac{1}{4}\right]$ and supp $m \subseteq\left[-\frac{1}{2}, \frac{1}{2}\right]$. Define $m_{l}(\xi)=m(\xi-l)$. Observe $m_{l}$ 's are normalized functions. So, we can get bilinear Carleson's theorem on $\mathbb{T}$ for $p_{3}>4 / 3$ from Theorem 2.2 with the above choice of $m_{l}$ by using transference techniques discussed above. Combining this with earlier observation for $1 \leq p_{3} \leq 2$ we have the following. 
Theorem 2.3. For $2 \leq p_{1}, p_{2} \leq \infty$ satisfying $\frac{1}{p_{1}}+\frac{1}{p_{2}}=\frac{1}{p_{3}}$, there is a constant $c$ such that for all functions $f, g \in \mathcal{P}(\mathbb{T})$

$$
\left\|\left(\sum_{l \in \mathbb{Z}}\left|\tilde{S}_{l}(f, g)\right|^{2}\right)^{\frac{1}{2}}\right\|_{p_{3}} \leq c\|f\|_{p_{1}}\|g\|_{p_{2}},
$$

where $\tilde{S}_{l}$ is the bilinear multiplier operator on $\mathbb{T}$ associated with $\chi_{\tilde{I}_{l}}$ for $\tilde{I}_{l}=[l, l+1), l \in \mathbb{Z}$.

As in the case of $\mathbb{R}$, we also show that for the above inequality (19) to hold, $p_{1}, p_{2} \geq 2$ is a necessary condition.

Proposition 2.4. For the conclusion of Theorem 2.3 to hold; $p_{1}, p_{2} \geq 2$ is a necessary condition.

Proof: Let $\tilde{I}_{l}=[l, l+1)$. Then for $g \equiv 1$ we have

$$
\begin{aligned}
\tilde{S}_{l}(f, g)(x) & =\sum_{n, m} \hat{f}(n) \hat{g}(m) \chi_{\tilde{I}_{l}}(n-m) e^{2 \pi i x(n+m)} \\
& =\hat{f}(l) e^{2 \pi i l x} .
\end{aligned}
$$

This implies that for $x \in \mathbb{T}$, we have

$$
\tilde{S}(f, g)(x)=\|f\|_{2}
$$

Assuming the inequality (19), we get

$$
\|f\|_{2} \leq c\|f\|_{p_{1}} \text {. }
$$

This would imply that $2 \leq p_{1} \leq \infty$. Similarly, by changing the role of $f$ and $g$ we get that $p_{2}$ also satisfies $2 \leq p_{2} \leq \infty$.

Thus the first issue has been resolved. Now for the second issue we apply transference techniques to the following result of Bernicot on $\mathbb{R}$.

Theorem $2.5([\mathbf{1}])$. Let $I_{l}=\left[a_{l}, b_{l}\right]$ be a sequence of disjoint intervals in $\mathbb{R}$ with $b_{l}-a_{l}=b_{l-1}-a_{l-1}$ and $a_{l+1}-b_{l}=a_{l}-b_{l-1}$ for all $l \in \mathbb{Z}$. Then for exponents $2<p_{1}, p_{2}, p_{3}^{\prime}<\infty$ satisfying $\frac{1}{p_{1}}+\frac{1}{p_{2}}=\frac{1}{p_{3}}$, there is a constant $C=C\left(p_{1}, p_{2}, p_{3}\right)$ such that for all functions $f, g \in \mathcal{S}(\mathbb{R})$

$$
\left\|\left(\sum_{l \in \mathbb{Z}}\left|S_{l}(f, g)\right|^{2}\right)^{\frac{1}{2}}\right\|_{p_{3}} \leq C\|f\|_{p_{1}}\|g\|_{p_{2}} .
$$


Observe that here symbols $\chi_{I_{l}}$, where $I_{l}$ 's are intervals, are not normalized functions. Nevertheless, by considering following modification we can treat them as normalized functions. Consider intervals $I_{l}=$ $\left(a_{l}-\frac{1}{4}, b_{l}+\frac{1}{4}\right) \subseteq \mathbb{R}$ and functions $\widehat{k_{l}}$ on $\mathbb{R}$ defined as follows

$$
\widehat{k_{l}}(\xi)= \begin{cases}1, & \xi \in I_{l} \\ 1 / 2, & \xi=a_{l}-\frac{1}{4}, b_{l}+\frac{1}{4} \\ 0, & \text { elsewhere. }\end{cases}
$$

Hence using the same arguments as earlier we get the following result.

Theorem 2.6. For $2<p_{1}, p_{2}, p_{3}^{\prime}<\infty$ satisfying $\frac{1}{p_{1}}+\frac{1}{p_{2}}=\frac{1}{p_{3}}$, where $p_{3}^{\prime}$ is the conjugate index of $p_{3}$, there is a constant $C=C\left(p_{1}, p_{2}, p_{3}\right)$ such that for all functions $f, g \in \mathcal{P}(\mathbb{T})$

$$
\left\|\left(\sum_{l \in \mathbb{Z}}\left|\tilde{S}_{l}(f, g)\right|^{2}\right)^{\frac{1}{2}}\right\|_{p_{3}} \leq C\|f\|_{p_{1}}\|g\|_{p_{2}},
$$

where the intervals $\tilde{I}_{l}$ satisfy the same hypothesis as in Theorem 2.5.

\section{Relation between $B^{p}(\mathbb{R})$ and $L^{p}(\mathbb{R})$ multipliers}

In this section we address the bilinear analogue of de Leeuw's result which establishes the connection between $L^{p}(\mathbb{R})$ multipliers and $B^{p}(\mathbb{R})$ multipliers. Our method of the proof basically lies in obtaining pointwise estimates for both the operators. For this we will use the ideas developed in $[\mathbf{1 1}]$, adapted to our setting. We would like to refer the reader to $[\mathbf{4}],[\mathbf{2}],[\mathbf{3}],[\mathbf{8}]$ for some work related to transference techniques in the bilinear settings.

We begin by considering the following lemmas which will be used to prove the main result of this section. The first lemma is an analogue of $[\mathbf{1 9}$, Lemma 3.9, p. 261] for almost periodic functions, while the second lemma tells us that for given an $L^{p}(\mathbb{R})$ function we can associate with it some periodic functions whose $B^{p}(\mathbb{R})$ norms and the $L^{p}(\mathbb{R})$ norms are related.

Lemma 3.1. For $f \in B^{\infty}(\mathbb{R})$, we have

$$
\lim _{\epsilon \rightarrow 0} \epsilon \int_{\mathbb{R}} f(x) G_{1, \epsilon}(x) d x=\lim _{T \rightarrow \infty} \frac{1}{2 T} \int_{-T}^{T} f(x) d x,
$$

where $G_{1, \epsilon}(x)=e^{-\pi \epsilon^{2} x^{2}}$. 
Proof: The proof follows using the same arguments as in $[\mathbf{1 9}$, Lemma 3.9, p. 261] along with the fact that trigonometric polynomials are dense in $B^{\infty}(\mathbb{R})$.

As an easy application of the above lemma we have the following observation which will be needed at a later stage.

Lemma 3.2. Let $P$ be a trigonometric polynomial. For $0<p, \epsilon<\infty$, define $f_{\epsilon}(x)=P(x) G_{p, \epsilon}(x)$, where $G_{p, \epsilon}(x)=e^{-\frac{\pi \epsilon^{2} x^{2}}{p}}$, then we have

$$
\lim _{\epsilon \rightarrow 0} \epsilon^{\frac{1}{p}}\left\|f_{\epsilon}\right\|_{L^{p}(\mathbb{R})}=\|P\|_{B^{p}(\mathbb{R})} .
$$

Proof: For the trigonometric polynomial $P$ and $0<p, \epsilon<\infty$, note that

$$
\begin{aligned}
\left\|f_{\epsilon}\right\|_{L^{p}(\mathbb{R})}^{p} & =\int_{\mathbb{R}}\left|P(x) G_{p, \epsilon}(x)\right|^{p} d x \\
& =\int_{\mathbb{R}}|P(x)|^{p} G_{1, \epsilon}(x) d x .
\end{aligned}
$$

Hence applying the Lemma 3.1 to the function $|P(x)|^{p}$, we obtain the desired result.

Lemma 3.3. Let $f \in L^{p}(\mathbb{R}), 0<p<\infty$. For $N \in \mathbb{N}$, let $f_{N}$ denote the truncated function $f \chi_{[-N / 2, N / 2]}$. Consider the $N$ periodic extension of $f_{N}$ given by $f_{N}^{\sharp}(x)=\sum_{n \in \mathbb{Z}} f_{N}(x+n N)$. Then

$$
\|f\|_{L^{p}(\mathbb{R})}=\lim _{N \rightarrow \infty} N^{\frac{1}{p}}\left\|f_{N}^{\sharp}\right\|_{B^{p}(\mathbb{R})} .
$$

Proof: Consider

$$
\begin{aligned}
\left\|f_{N}^{\sharp}\right\|_{B^{p}(\mathbb{R})} & =\lim _{T \rightarrow \infty}\left(\frac{1}{2 T} \int_{-T}^{T}\left|f_{N}^{\sharp}(x)\right|^{p} d x\right)^{\frac{1}{p}} \\
& =\lim _{k \rightarrow \infty}\left(\frac{1}{k N} \sum_{i=0}^{k-1} \int_{(-k+2 i) \frac{N}{2}}^{(-k+2(i+1)) \frac{N}{2}}\left|f_{N}^{\sharp}(x)\right|^{p} d x\right)^{\frac{1}{p}} \\
& =N^{-\frac{1}{p}}\left\|f_{N}\right\|_{L^{p}(\mathbb{R})} .
\end{aligned}
$$

Thus we obtain the desired result.

Now we are in position to prove the main result of this section.

Theorem 3.4. Let $m(t, s)$ be a bounded continuous function. Then $m \in M_{p_{1}, p_{2}}^{p_{3}}(\mathbb{R})$ if and only if $m \in \mathcal{M}_{p_{1}, p_{2}}^{p_{3}}(\mathbb{R})$, where $1 \leq p_{1}, p_{2} \leq \infty$ with $\frac{1}{p_{1}}+\frac{1}{p_{2}}=\frac{1}{p_{3}}$ and the values of $p_{3}$ could be lower or bigger than one. 
Proof: Let $e_{\lambda_{i}}(x)=e^{2 \pi i \lambda_{i} x}, \lambda_{i} \in \mathbb{R}, i=1,2$ and set $f_{i, \epsilon}(x)=e_{\lambda_{i}}(x) G_{p_{i}, \epsilon}(x)$, where $G_{p_{i}, \epsilon}(x)=e^{-\frac{\pi \epsilon^{2} x^{2}}{p_{i}}}$. Note that $\hat{f}_{i, \epsilon}(\xi)=\sqrt{p_{i}} \epsilon^{-1} e^{-\pi p_{i} \frac{\left(\xi-\lambda_{i}\right)^{2}}{\epsilon^{2}}}$. Now consider

$$
\begin{aligned}
& T\left(f_{1, \epsilon}, f_{2, \epsilon}\right)(x)= \int_{\mathbb{R}} \int_{\mathbb{R}} \hat{f}_{1, \epsilon}(\xi) \hat{f}_{2, \epsilon}(\eta) m(\xi, \eta) e^{2 \pi i x(\xi+\eta)} d \xi d \eta \\
&= \sqrt{p_{1} p_{2}} \epsilon^{-2} \int_{\mathbb{R}} \int_{\mathbb{R}} e^{-\pi p_{1} \frac{\left(\xi-\lambda_{1}\right)^{2}}{\epsilon^{2}}} e^{-\pi p_{2} \frac{\left(\eta-\lambda_{2}\right)^{2}}{\epsilon^{2}}} \\
& \times m(\xi, \eta) e^{2 \pi i x(\xi+\eta)} d \xi d \eta,
\end{aligned}
$$

and

$$
B\left(e_{\lambda_{1}}, e_{\lambda_{2}}\right)(x)=m\left(\lambda_{1}, \lambda_{2}\right) e^{2 \pi i x\left(\lambda_{1}+\lambda_{2}\right)} .
$$

We shall estimate the following.

$$
\begin{aligned}
B\left(e_{\lambda_{1}}, e_{\lambda_{2}}\right)(x) G_{p_{3}, \epsilon}(x)-T\left(f_{1, \epsilon}, f_{2, \epsilon}\right)(x) & \\
= & m\left(\lambda_{1}, \lambda_{2}\right) e^{2 \pi i x \lambda_{1}} e^{-\frac{\pi \epsilon^{2} x^{2}}{p_{1}}} e^{2 \pi i x \lambda_{2}} e^{-\frac{\pi \epsilon^{2} x^{2}}{p_{2}}}-T\left(f_{1, \epsilon}, f_{2, \epsilon}\right)(x) \\
= & \sqrt{p_{1} p_{2}} \epsilon^{-2} \int_{\mathbb{R}} \int_{\mathbb{R}}\left(m\left(\lambda_{1}, \lambda_{2}\right)-m(\xi, \eta)\right) \\
\times & \times e^{-\pi p_{1} \frac{\left(\xi-\lambda_{1}\right)^{2}}{\epsilon^{2}}} e^{-\pi p_{2} \frac{\left(\eta-\lambda_{2}\right)^{2}}{\epsilon^{2}}} e^{2 \pi i x(\xi+\eta)} d \xi d \eta \\
= & \sqrt{p_{1} p_{2}} \int_{\mathbb{R}} \int_{\mathbb{R}}\left(m\left(\lambda_{1}, \lambda_{2}\right)-m\left(\epsilon \xi+\lambda_{1}, \epsilon \eta+\lambda_{2}\right)\right) \\
\times & \times e^{-\pi p_{1} \xi^{2}} e^{-\pi p_{2} \eta^{2}} e^{2 \pi i x\left(\epsilon \xi+\lambda_{1}+\epsilon \eta+\lambda_{2}\right)} d \xi d \eta .
\end{aligned}
$$

Using the Lebesgue dominated convergence theorem we obtain

$$
\lim _{\epsilon \rightarrow 0}\left\|B\left(e_{\lambda_{1}}, e_{\lambda_{2}}\right)(x) G_{p_{3}, \epsilon}(x)-T\left(f_{1, \epsilon}, f_{2, \epsilon}\right)(x)\right\|_{\infty}=0 .
$$

In fact the above estimate is valid for general trigonometric polynomials $P_{i}, i=1,2$ and in this case the functions $f_{i, \epsilon}$ are given by $f_{i, \epsilon}(x)=P_{i}(x) G_{p_{i}, \epsilon}(x), i=1,2$. Using (22) we have

$$
\begin{aligned}
\left\|B\left(P_{1}, P_{2}\right)\right\|_{B^{p_{3}(\mathbb{R})}}^{p_{3}} & =\lim _{\epsilon \rightarrow 0} \epsilon \int_{\mathbb{R}}\left|B\left(P_{1}, P_{2}\right)(x) G_{p_{3}, \epsilon}(x)\right|^{p_{3}} d x \\
& =\lim _{\epsilon \rightarrow 0} \int_{\mathbb{R}}\left|B\left(P_{1}, P_{2}\right)(x / \epsilon) G_{p_{3}, 1}(x)\right|^{p_{3}} d x .
\end{aligned}
$$


We write the right hand side as $J$ and for $R>0$ which will be chosen to be large enough later, we decompose it into $J_{R}$ and $J^{R}$ where,

$$
J_{R}=\lim _{\epsilon \rightarrow 0} \int_{|x|<R}\left|B\left(P_{1}, P_{2}\right)(x / \epsilon) G_{p_{3}, 1}(x)\right|^{p_{3}} d x
$$

and

$$
J^{R}=\lim _{\epsilon \rightarrow 0} \int_{|x|>R}\left|B\left(P_{1}, P_{2}\right)(x / \epsilon) G_{p_{3}, 1}(x)\right|^{p_{3}} d x .
$$

Now we will estimate $J_{R}$ and $J^{R}$ separately. Consider

$$
\begin{aligned}
J^{R} & =\lim _{\epsilon \rightarrow 0} \int_{|x|>R}\left|B\left(P_{1}, P_{2}\right)(x / \epsilon) G_{p_{3}, 1}(x)\right|^{p_{3}} d x \\
& \leq M \int_{|x|>R}\left(G_{p_{3}, 1}(x)\right)^{p_{3}} d x,
\end{aligned}
$$

where $M=\left\|B\left(P_{1}, P_{2}\right)\right\|_{\infty}$. For fixed $\delta>0$ choose $R$ large enough say $R_{0}$, such that.

$$
J^{R_{0}} \leq \frac{\delta}{2}
$$

Now consider

$$
\begin{aligned}
J_{R_{0}}= & \lim _{\epsilon \rightarrow 0} \int_{|x|<R_{0}}\left|B\left(P_{1}, P_{2}\right)(x / \epsilon) G_{p_{3}, 1}(x)\right|^{p_{3}} d x \\
= & \lim _{\epsilon \rightarrow 0} \epsilon \int_{|x|<\frac{R_{0}}{\epsilon}}\left|B\left(P_{1}, P_{2}\right)(x) G_{p_{3}, \epsilon}(x)\right|^{p_{3}} d x \\
\leq & \lim _{\epsilon \rightarrow 0} \epsilon \int_{|x|<\frac{R_{0}}{\epsilon}}\left|B\left(P_{1}, P_{2}\right)(x) G_{p_{3}, \epsilon}(x)-T\left(f_{1, \epsilon}, f_{2, \epsilon}\right)(x)\right|^{p_{3}} d x \\
& +\lim _{\epsilon \rightarrow 0} \epsilon \int_{|x|<\frac{R_{0}}{\epsilon}}\left|T\left(f_{1, \epsilon}, f_{2, \epsilon}\right)(x)\right|^{p_{3}} d x \\
\leq & R_{0} \lim _{\epsilon \rightarrow 0}\left\|B\left(P_{1}, P_{2}\right)(.) G_{p_{3}, \epsilon}(x)-T\left(f_{1, \epsilon}, f_{2, \epsilon}\right)(.)\right\|_{\infty}^{p_{3}} \\
& +\lim _{\epsilon \rightarrow 0} \epsilon \int_{\mathbb{R}}\left|T\left(f_{1, \epsilon}, f_{2, \epsilon}\right)(x)\right|^{p_{3}} d x .
\end{aligned}
$$

Here we have used that $p_{3}<1$. For $p_{3} \geq 1$ also, it can be done with a constant depending on $p_{3}$. Using (25) we obtain,

$$
J_{R_{0}} \leq \frac{\delta}{2}+\lim _{\epsilon \rightarrow 0} \epsilon \int_{\mathbb{R}}\left|T\left(f_{1, \epsilon}, f_{2, \epsilon}\right)(x)\right|^{p_{3}} d x .
$$


As $\delta$ is arbitrarily small, putting all this together we obtain

$$
\begin{aligned}
\left\|B\left(P_{1}, P_{2}\right)\right\|_{B^{p_{3}}(\mathbb{R})} & \leq \lim _{\epsilon \rightarrow 0} \epsilon^{\frac{1}{p_{3}}}\left\|T\left(f_{1, \epsilon}, f_{2, \epsilon}\right)(x)\right\|_{p_{3}} \\
& \leq C \lim _{\epsilon \rightarrow 0} \epsilon^{\frac{1}{p_{1}}}\left\|f_{1, \epsilon}\right\|_{p_{1}} \epsilon^{\frac{1}{p_{2}}}\left\|f_{2, \epsilon}\right\|_{p_{2}} \\
& =C\left\|P_{1}\right\|_{B^{p_{1}}(\mathbb{R})}\left\|P_{2}\right\|_{B^{p_{2}}(\mathbb{R})} .
\end{aligned}
$$

Here in the last step we have used Lemma 3.2. This proves the sufficient part.

Converse. Let $f, g \in \mathcal{S}(\mathbb{R})$ be compactly supported functions. Without loss of generality we can assume that the supports of $f$ and $g$ are contained in $[-N / 2, N / 2]$ for some large $N \in \mathbb{N}$. Let $f_{N}^{\sharp}$ and $g_{N}^{\sharp}$ be the $N$ periodic extensions of $f$ and $g$ respectively. Note that $\hat{f}_{N}^{\sharp}$ and $\hat{g}_{N}^{\sharp}$ are supported in $\frac{1}{N} \mathbb{Z}$ and satisfy

$$
\begin{aligned}
\hat{f}_{N}^{\sharp} & \left(\frac{n}{N}\right)=\frac{1}{N} \hat{f}\left(\frac{n}{N}\right), \quad \hat{g}_{N}^{\sharp}\left(\frac{n}{N}\right)=\frac{1}{N} \hat{g}\left(\frac{n}{N}\right) . \\
T(f, g)(x) & =\int_{\mathbb{R}} \int_{\mathbb{R}} \hat{f}(\xi) \hat{g}(\eta) m(\xi, \eta) e^{2 \pi i x(\xi+\eta)} d \xi d \eta \\
& =\lim _{N \rightarrow \infty} \frac{1}{N^{2}} \sum_{n, k \in \mathbb{Z}} \hat{f}\left(\frac{n}{N}\right) \hat{g}\left(\frac{k}{N}\right) m\left(\frac{n}{N}, \frac{k}{N}\right) e^{2 \pi i x \frac{n+k}{N}} \\
& =\lim _{N \rightarrow \infty} \sum_{t, s} \hat{f}_{N}^{\sharp}(t) \hat{g}_{N}^{\sharp}(s) m(t, s) e^{2 \pi i x(t+s)} \\
& =\lim _{N \rightarrow \infty} B\left(f_{N}^{\sharp}, g_{N}^{\sharp}\right)(x) .
\end{aligned}
$$

Note that for each $N, B\left(f_{N}^{\sharp}, g_{N}^{\sharp}\right)$ is an $N$ periodic function. Hence for $0<p<\infty$ we have

$$
\left\|B\left(f_{N}^{\sharp}, g_{N}^{\sharp}\right)\right\|_{B(\mathbb{R})}^{p}=N^{-1} \int_{-N / 2}^{N / 2}\left|B\left(f_{N}^{\sharp}, g_{N}^{\sharp}\right)(x)\right|^{p} d x .
$$

It suffices to prove that for large $L>0$

$$
\int_{-L}^{L}|T(f, g)(x)|^{p_{3}} d x \leq C\|f\|_{p_{1}}^{p_{3}}\|g\|_{p_{2}}^{p_{3}}
$$


with a constant $C$ independent of the parameter $L$. Consider

$$
\begin{aligned}
\int_{-L}^{L}|T(f, g)(x)|^{p_{3}} d x & \leq \liminf _{N \rightarrow \infty} \int_{-L}^{L}\left|B\left(f_{N}^{\sharp}, g_{N}^{\sharp}\right)(x)\right|^{p_{3}} d x \\
& \leq \liminf _{N \rightarrow \infty} N\left\|B\left(f_{N}^{\sharp}, g_{N}^{\sharp}\right)\right\|_{B^{p_{3}}(\mathbb{R})}^{p_{3}} \\
& \leq C \liminf _{N \rightarrow \infty} N\left\|f_{N}^{\sharp}\right\|_{B^{p_{1}}(\mathbb{R})}^{p_{3}}\left\|g_{N}^{\sharp}\right\|_{B^{p_{2}}(\mathbb{R})}^{p_{3}} \\
& =C\|f\|_{p_{1}}^{p_{3}}\|g\|_{p_{2}}^{p_{3}} .
\end{aligned}
$$

Last equality is obtained by using Lemma 3.3. Hence the result follows.

\section{Restriction from $\mathbb{R}^{n}$ to $\mathbb{R}^{k}$}

In this section, we prove the bilinear analogue of a result due to de Leeuw, regarding restriction of multipliers from $\mathbb{R}^{n}$ to $\mathbb{R}^{k}$ for $k<n$. For $p_{3} \geq 1$, it has been proved by Diestel and Grafakos [8]. The authors used duality to prove their result, but for $p_{3}<1$ duality arguments are not permissible. We extend this result to the entire range of the exponents $p_{1}, p_{2}$ and $p_{3}$ by using a different idea.

Let $m\left(\xi_{1}, \xi_{2}, \ldots, \xi_{n}, \eta_{1}, \eta_{2}, \ldots, \eta_{n}\right)$ be a bounded continuous function defined on $\mathbb{R}^{n} \times \mathbb{R}^{n}$. Let $k<n$, for fixed $\xi_{k+1}, \ldots, \xi_{n}, \eta_{k+1}, \ldots, \eta_{n} \in \mathbb{R}$ consider the function $m_{k}\left(\xi_{1}, \ldots, \xi_{k}, \eta_{1}, \ldots, \eta_{k}\right)=m\left(\xi_{1}, \ldots, \xi_{n}, \eta_{1}, \ldots, \eta_{n}\right)$. Then we have the following theorem

Theorem 4.1. Let $m \in M_{p_{1}, p_{2}}^{p_{3}}\left(\mathbb{R}^{n}\right)$ be a continuous function. Then $m_{k} \in M_{p_{1}, p_{2}}^{p_{3}}\left(\mathbb{R}^{k}\right)$ defined as above. Moreover, $\left\|m_{k}\right\| \leq\|m\|$.

To avoid notational complications, we will prove the above theorem for $n=2$ and $k=1$. The general case follows similarly. We will first prove the following lemma.

Lemma 4.2. Let $m\left(\xi_{1}, \xi_{2}, \eta_{1}, \eta_{2}\right) \in M_{p_{1}, p_{2}}^{p_{3}}\left(\mathbb{R}^{2}\right)$. For $\xi_{0}, \eta_{0} \in \mathbb{R}$ and $t>0$ consider the functions $m_{0}\left(\xi_{1}, \xi_{2}, \eta_{1}, \eta_{2}\right)=m\left(\xi_{1}, \xi_{0}+\xi_{2}, \eta_{1}, \eta_{0}+\eta_{2}\right)$ and $m_{t}\left(\xi_{1}, \xi_{2}, \eta_{1}, \eta_{2}\right)=m\left(\xi_{1}, t \xi_{2}, \eta_{1}, t \eta_{2}\right)$. Then $m_{0}, m_{t} \in M_{p_{1}, p_{2}}^{p_{3}}\left(\mathbb{R}^{2}\right)$ with $\left\|m_{0}\right\|=\left\|m_{t}\right\|=\|m\|$.

Proof: Let $T_{0}, T_{t}$ be the operators associated with $m_{0}$ and $m_{t}$ respectively. Note that for $f, g \in \mathcal{S}\left(\mathbb{R}^{2}\right)$,

$$
T_{0}(f, g)(x, y)=e^{2 \pi i y\left(\xi_{0}+\eta_{0}\right)} T\left(M_{0, \xi_{0}} f, M_{0, \eta_{0}} g\right)(x, y),
$$


where $M_{\xi, \eta} f(x, y)=e^{2 \pi i(x \xi+y \eta)} f(x, y)$ and

$$
T_{t}(f, g)(x, y)=T\left(D_{1, t} f, D_{1, t} g\right)(x, y / t),
$$

where $D_{s, t} f(x, y)=f(s x, t y)$.

Hence the lemma follows.

Proof of Theorem 4.1: For fixed $\xi_{0}, \eta_{0} \in \mathbb{R}$, write $\xi=\left(\xi_{1}, \xi_{0}\right)$ and $\eta=$ $\left(\eta_{1}, \eta_{0}\right)$. For $f, g \in \mathcal{S}(\mathbb{R})$ consider

$$
\begin{aligned}
& \left\|T_{m_{1}}(f, g)\right\|_{p_{3}}^{p_{3}} \\
& \quad=\int_{\mathbb{R}}\left|\int_{\mathbb{R}^{2}} \hat{f}\left(\xi_{1}\right) \hat{g}\left(\eta_{1}\right) m(\xi, \eta) e^{2 \pi i x\left(\xi_{1}+\eta_{1}\right)} d \xi_{1} d \eta_{1}\right|^{p_{3}} d x \\
& =\pi^{-\frac{1}{2}} \int_{\mathbb{R}^{2}} e^{-\pi y^{2}}\left|\int_{\mathbb{R}^{2}} \hat{f}\left(\xi_{1}\right) \hat{g}\left(\eta_{1}\right) m(\xi, \eta) e^{2 \pi i x\left(\xi_{1}+\eta_{1}\right)} d \xi_{1} d \eta_{1}\right|^{p_{3}} d x d y \\
& =\pi^{-\frac{1}{2}} \int_{\mathbb{R}^{2}}\left|\int_{\mathbb{R}^{2}} \hat{f}\left(\xi_{1}\right) \hat{g}\left(\eta_{1}\right) m(\xi, \eta) e^{2 \pi i x\left(\xi_{1}+\eta_{1}\right)} e^{\frac{-\pi y^{2}}{p_{3}}} d \xi_{1} d \eta_{1}\right|^{p_{3}} d x d y .
\end{aligned}
$$

Let $F(x, y)=f(x) e^{\frac{-\pi y^{2}}{p_{1}}}$ and $G(x, y)=g(x) e^{\frac{-\pi y^{2}}{p_{2}}}$, then

$$
\begin{aligned}
\left\|T_{m_{1}}(f, g)\right\|_{p_{3}}^{p_{3}} \leq \pi^{-\frac{1}{2}} \lim _{t \rightarrow 0} \int_{\mathbb{R}^{2}} \mid \int_{\mathbb{R}^{4}} \hat{F}\left(\xi_{1}, \xi_{2}\right) \hat{G}\left(\eta_{1}, \eta_{2}\right) \\
\quad \times\left. m_{0, t}(\xi, \eta) e^{2 \pi i\left(x\left(\xi_{1}+\eta_{1}\right)+y\left(\xi_{2}+\eta_{2}\right)\right)} d \bar{\xi} d \bar{\eta}\right|^{p_{3}} d x d y,
\end{aligned}
$$

where $m_{0, t}(\xi, \eta)=m\left(\xi_{1}, \xi_{0}+t \xi_{2} ; \eta_{1}, \eta_{0}+t \eta_{2}\right)$ and $d \bar{\xi} d \bar{\eta}=d \xi_{1} d \xi_{2} d \eta_{1} d \eta_{2}$.

Using Lemma 4.2, we see that $m_{0, t} \in M_{p_{1}, p_{2}}^{p_{3}}\left(\mathbb{R}^{2}\right)$ and $\left\|m_{0, t}\right\|=\|m\|$.

$$
\left\|T_{m_{1}}(f, g)\right\|_{p_{3}}^{p_{3}} \leq \pi^{-\frac{1}{2}}\|m\|^{p_{3}}\|F\|_{p_{1}}^{p_{3}}\|G\|_{p_{2}}^{p_{3}} .
$$

This completes the proof.

\section{References}

[1] F. BeRnicot, $L^{p}$ estimates for non-smooth bilinear Littlewood-Paley square functions on $\mathbb{R}$, Math. Ann. (to appear).

[2] O. Blasco, Bilinear multipliers and transference, Int. J. Math. Math. Sci. 4 (2005), 545-554.

[3] O. Blasco, M. Carro, and T. A. Gillespie, Bilinear Hilbert transform on measure spaces, J. Fourier Anal. Appl. 11(4) (2005), $459-470$. 
[4] D. Bose, S. Madan, P. Mohanty, and S. Shrivastava, Relations between bilinear multipliers on $\mathbb{R}^{n}, \mathbb{T}^{n}$ and $\mathbb{Z}^{n}$, Proc. Indian Acad. Sci. Math. Sci. 119(4) (2009), 501-512.

[5] L. Carleson, On the Littlewood-Paley Theorem, Inst. MittagLeffler, Report (1967).

[6] R. R. Coifman And G. Weiss, "Transference methods in analysis", Conference Board of the Mathematical Sciences Regional Conference Series in Mathematics 31, American Mathematical Society, Providence, R.I., 1976.

[7] K. DE Leeuw, On $L_{p}$ multipliers, Ann. of Math. (2) 81 (1965), $364-379$.

[8] G. Diestel and L. Grafakos, Unboundedness of the ball bilinear multiplier operator, Nagoya Math. J. 185 (2007), 151-159.

[9] T. A. Gillespie And J. L. Torrea, Transference of a LittlewoodPaley-Rubio inequality and dimension free estimates, Rev. Un. Mat. Argentina 45(1) (2004), 1-6 (2005).

[10] L. Grafakos, "Classical and modern Fourier analysis", Pearson Education, Inc., Upper Saddle River, NJ, 2004.

[11] L. Grafakos And P. Honzík, Maximal transference and summability of multilinear Fourier series, J. Aust. Math. Soc. 80(1) (2006), 65-80.

[12] L. Grafakos and R. H. Torres, Multilinear Calderón-Zygmund theory, Adv. Math. 165(1) (2002), 124-164.

[13] M. T. LACEY, On bilinear Littlewood-Paley square functions, Publ. Mat. 40(2) (1996), 387-396.

[14] M. Lacey And C. Thiele, $L^{p}$ estimates on the bilinear Hilbert transform for $2<p<\infty$, Ann. of Math. (2) 146(3) (1997), 693-724.

[15] M. Lacey And C. Thiele, On Calderón's conjecture, Ann. of Math. (2) 149(2) (1999), 475-496.

[16] S. Madan And P. Mohanty, Jodeit's extensions for bilinear multipliers, Bull. Lond. Math. Soc. 40(6) (2008), 937-944.

[17] P. Mohanty and S. Shrivastava, A note on the bilinear Littlewood-Paley square function, Proc. Amer. Math. Soc. 138(6) (2010), 2095-2098.

[18] J. L. Rubio DE Francia, A Littlewood-Paley inequality for arbitrary intervals, Rev. Mat. Iberoamericana 1(2) (1985), 1-14.

[19] E. M. Stein AND G. Weiss, "Introduction to Fourier analysis on Euclidean spaces", Princeton Mathematical Series 32, Princeton University Press, Princeton, N.J., 1971. 
Department of Mathematics and Statistics Indian Institute of Technology

Kanpur - 208016

India

E-mail address: parasar@iitk.ac.in

E-mail address: saurabhk@iitk.ac.in

Primera versió rebuda el 4 d'octubre de 2010, darrera versió rebuda el 3 de desembre de 2010. 